\title{
Atelier de production écrite en 1ère année du cycle
} 2

Jacquy Albert

\section{OpenEdition}

1 Journals

Édition électronique

URL : http://journals.openedition.org/trema/2256

DOI : 10.4000/trema.2256

ISSN : 2107-0997

Éditeur

Faculté d'Éducation de l'université de Montpellier

\section{Édition imprimée}

Date de publication : 1 décembre 1994

Pagination : 69-78

ISSN : 1167-315X

\section{Référence électronique}

Jacquy Albert, « Atelier de production écrite en 1ère année du cycle 2 », Tréma [En ligne], 6 | 1994, mis en ligne le 01 décembre 1994, consulté le 21 décembre 2020. URL : http://journals.openedition.org/ trema/2256 ; DOI : https://doi.org/10.4000/trema.2256

Ce document a été généré automatiquement le 21 décembre 2020.

Trema 


\title{
Atelier de production écrite en lère année du cycle 2
}

\author{
Jacquy Albert
}

1 A la rentrée scolaire 1989, l'équipe universitaire de recherche en éducation et didactique (E.U.R.E.D.) de l'université de Toulouse-le-Mirail a mis en œuvre, dans le département de l'Aude, une recherche-action visant à développer une démarche innovante de l'acquisition de la langue écrite. Depuis cette date, près d'une centaine de formateurs et d'enseignants de cycle 2 se sont impliqués dans cette démarche. Devant l'importante demande manifestée par le corps enseignant, les stages de formation ont été ouverts au maximum. De sorte qu'aujourd'hui, la population concernée peut être estimée à plus de 1200 élèves et est tout à fait hétérogène. Parce qu'il s'agit d'une recherche-action, expérimentation et pratique, intimement liées, constituent un ensemble en évolution permanente. Cette démarche, à différencier d'une "méthode de lecture" au sens où cette expression est habituellement comprise, présente des approches multivariées. En effet, il s'agit plus d'apporter de nouveaux points de vue, à travers des techniques pédagogiques précises et organisées, que de donner un programme de travail. L'enfant placé au centre $d u$ système éducatif est porteur d'un savoir qu'il doit pouvoir exprimer et qui servira de base à la construction de la langue écrite dans un contexte de communication. La mise en œuvre de cette idée générale s'appuie sur les objectifs opérationnels suivants :

1. L'enfant doit prendre plaisir à entrer dans le langage écrit.

2. L'enfant doit acquérir une certaine autonomie et apprendre avec les autres.

3. L'enfant doit construire son apprentissage à partir de son langage oral.

4. L'enfant doit faire l'acquisition simultanée de la lecture et de l'écriture.

2 Dans le cadre de cette démarche, nous avons instauré une structure particulière de travail appelée "la roue", dont nous présenterons ici une application.

Cette séquence dure une heure, la classe est organisée en ateliers. Chaque groupe (quatre enfants par groupe) va passer à tour de rôle dans chaque atelier. La durée de travail dans un atelier est de dix minutes. Le nom de cette séquence ("la roue") vient du dispositif 
matériel adopté pour faciliter le repérage des enfants. Il s'agit de deux roues concentriques, l'une de cinquante centimètres de diamètre fixe, et l'autre mobile de vingt-cinq centimètres. Elles sont partagées en secteurs (un secteur pour chaque groupe d'enfants). Sur la plus grande sont écrits les noms de chaque atelier, sur la plus petite sont collées des pastilles de couleur qui désignent les groupes d'enfants.

4 A la fin de chaque séquence, la maîtresse signale qu'il faut changer d'atelier et tourne la roue. Les enfants savent où ils doivent aller par un simple regard sur la roue.

Les enfants sont regroupés par affinité à l'aide d'une épreuve sociométrique. Ces groupes sont durables mais peuvent être modifiés aisément.

6 Quatre ateliers sont "autonomes" : les enfants sont seuls pour effectuer leur tâche. Le cinquième atelier est l'atelier "d'accompagnement". C'est là que l'enseignante va travailler avec les enfants. La règle fondamentale est fixée dès la mise en place de ce travail : les élèves qui sont dans les ateliers autonomes n'ont pas le droit de déranger la maîtresse.

7 Nous ne présenterons et commenterons, ici, qu'une série de trois ateliers illustrant une séquence d'écriture productive.

\section{Atelier autonome}

\section{Objectifs :}

1. Mettre les enfants en situation de production d'écrit.

2. Leur permettre d'utiliser leur savoir dans cette tâche.

3. Les inciter à chercher tous les indices dont ils disposent.

4. Echanger leurs connaissances.

5. Permettre à l'enseignant d'évaluer le niveau de connaissance des enfants.

9 Durée : dix minutes.

10 Fréquence : en fonction du moment de l'année et du besoin en textes de la classe (Cette activité devient de plus en plus fréquente en approchant de la fin de l'année). Une fois par quinzaine, puis une fois par semaine semblent être un bon rythme.

11 Matériel : une demi-feuille de papier par enfant, des pochettes de feutres de couleur, des crayons gris.

12 Consignes : chaque enfant du groupe fait un dessin puis en écrit la légende.

\section{Commentaires}

13 L'atelier est autonome. C'est dire que l'enfant n'a pas le droit de déranger la maitresse qui est, pendant ce temps, occupée avec d'autres enfants dans l'atelier d'accompagnement. Par contre, il a trois compagnons avec qui il peut communiquer librement. Eux aussi ont à effectuer une tâche semblable. C'est un point remarquable de cette activité, que de mettre ensemble des enfants, face à une forme identique de problèmes à résoudre. Le contenu de l'écrit étant déterminé par leur dessin, ils sont donc en situation de communiquer sur la façon de résoudre le problème, sans pouvoir simplement copier le voisin. Il est vrai que le cas de la simple copie se produit aussi, mais cela a tendance à s'atténuer à mesure qu'augmente la confiance en soi de l'enfant et son désir d'apporter une production originale. 

enfants tout en diminuant leur niveau de dépendance par rapport à l'adulte. Il est, en plus, un lieu de production d'écrits où l'enfant va mettre en oeuvre son savoir sur la langue écrite. Ce savoir sera accepté et utilisé par la maîtresse, l'enfant le sait. On obtient, bien sûr, des productions tout à fait différentes en fonction des enfants et du moment de l'année. Ainsi, Ozlem écrit, en janvier :

\section{PLETZISA UD H BKROC}

pour raconter son histoire. Elle sait que l'écriture est constituée d'une suite de signes conventionnels qu'il faut tracer de gauche à droite en suivant une ligne. Elle utilise préférentiellement des lettres contenues dans son prénom $(\mathrm{O}, \mathrm{L}, \mathrm{E}, \mathrm{Z})$, que nous retrouvons au début de sa production, puis elle a cherché dans les ressources qui sont à sa disposition, d'autres lettres, pour obtenir une variété plus grande. Malgré la fréquentation assidue de l'écrit, Ozlem n'a pas encore intégré l'organisation de la phrase en mots même si elle sait les désigner dans les textes. A la même période de l'année, Jordan a écrit : JE SUIS ALLE AUX POMMES, en utilisant un savoir tout à fait différent.
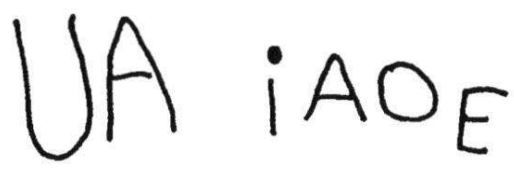

D'abord il sait que la chaîne écrite présente des segmentations, il faut tracer des groupes de lettres pour écrire. Mais il y a beaucoup plus dans sa production. Elle montre qu'il a compris que l'écriture est une traduction de ce qui est dit. Il connaît la valeur sonore de certaines lettres (de combien ?) et s'en sert.

Je suis allé aux pommes.
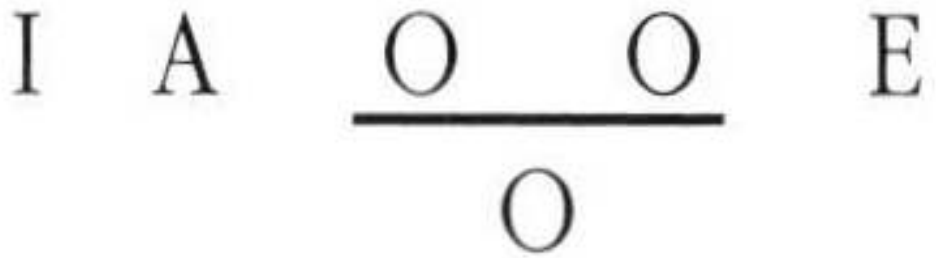

Son écriture montre bien la dimension parcellaire de son savoir et les confusions qui existent dans son esprit. Pour lui les mots sont un ensemble de lettres, et les lettres écrivent ce qu'on dit. Pour écrire son texte, Jordan a utilisé son savoir : il est déjà avancé dans son analyse.

Toujours au même moment de l'année, Yannick propose :

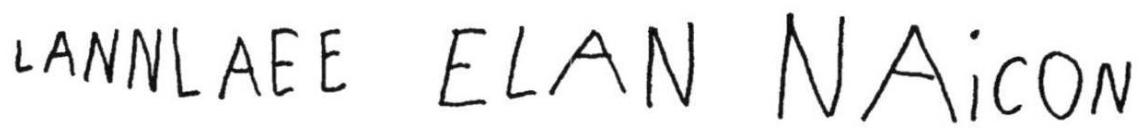


pour... L'ARBRE EST TRANQUILLE.

LA FILLE P(rend) DES POMMES. repérable.

L'arbre est tranquille.

\section{LA EE A I} possible. chez cet enfant. des questions.

En avril, Ozlem peut écrire :

Comme dans le texte d'Ozlem, on remarque que les lettres de son prénom apparaissent au début de son histoire. Il est clair que les lettres contenues dans le prénom des enfants ont un statut privilégié. Sans doute est-ce dû au fait que le prénom est la première écriture reconnue produite par l'enfant, et qu'il la répète souvent. On notera aussi que la segmentation en mots est respectée et que la valeur sonore de quelques lettres est

Pourquoi le mot "est" traduit par "ELAN" a-t-il pris cette forme ? Yannick a-t-il repéré ce mot, dans un livre ou un texte, parce qu'il commence par la même lettre? Cela est

Mais ces savoirs sont tout à fait incertains pour l'enfant qui n'a pas entendu le L de "tranquille" comme celui de "L'arbre" qui occupe une place privilégiée en début de phrase. De même, la segmentation en mots semble plus être le fait de cette phrase particulière où la segmentation à l'oral est relativement plus sensible que dans d'autres phrases. Il n'en demeure pas moins qu'un processus d'analyse de l'écrit est déjà à l'œuvre

Ces exemples montrent que les écrits des enfants, qui sont des productions hors norme dans la mesure où nous ne pouvons pas les lire, sont porteurs d'une partie de leurs connaissances. Cela montre aussi qu'ils ne font pas n'importe quoi et qu'il est utile, pour mieux les comprendre, de les considérer avec un regard positif. Les enfants savent que l'écrit résulte de l'application d'un code, mais ils n'en connaissent pas les règles. Ces règles, ils les construisent dans l'action. Il ne suffit pas de les leur dire, ils doivent les intégrer. Si Ozlem est tout à fait capable de montrer un mot dans un livre, comme elle n'a pas compris pourquoi cet ensemble de lettres est un mot, elle ne peut pas le produire. En lui donnant l'occasion de produire de l'écrit, nous lui permettons de se poser et de poser

\section{LA FILLE P LES POMMES.}

Maintenant elle sait que les mots ne s'écrivent pas avec n'importe quelles lettres, qu'ils ont une forme (presque) constante. Alors elle les cherche dans les ressources de la classe. Mais comme on ne trouve pas toujours tout ce qu'on cherche sur les murs, dans les cahiers, les livres, ou dans le dictionnaire de la classe, on doit encore se poser des questions pour écrire des mots nouveaux. Ozlem a bien trouvé le P de "prend", mais le reste est vraiment trop difficile. Elle le demandera à la maîtresse pendant l'atelier d'accompagnement. 
Tout est fait, ici, pour que l'enfant soit confronté au problème de la production d'écrits et pour qu'il utilise son savoir, celui de ses camarades et les ressources de l'environnement. Il doit chercher, choisir, prendre des décisions... la maîtresse, qui sait, n'étant pas accessible pour le moment.

Ce qui est important, dans cette séquence, n'est pas de faire écrire l'enfant sur un dessin ou une image, tous les enseignants le font. C'est de lui permettre de le faire en utilisant ce qu'il sait, sans en attendre une production normée. Nous voyons que l'attitude de l'enseignant est sans doute le point central de la réussite de l'activité.

\section{Atelier d'accompagnement}

\section{Objectifs :}

1. Favoriser l'expression des procédures utilisées par les enfants.

2. Evaluer leur niveau de compréhension du fonctionnement de la langue écrite.

3. Aider les enfants à découvrir des procédures de résolution des problèmes.

4. Utiliser les productions des enfants.

Durée : dix minutes par groupe.

Fréquence : quotidienne.

Matériel : dans le cas particulier de l'atelier d'accompagnement qui succède à l'atelier autonome décrit plus haut: les dessins et les textes produits par les enfants, papier, crayons.

\section{Commentaires}

Dans cet atelier, l'enseignant va apprendre beaucoup de l'enfant, car il lui demande de préciser et d'expliciter sa production.

$$
\text { DISE AU CHE RCHELESOE UFS. }
$$

C'est le moment où il va comprendre où en est l'enfant. C'est aussi le moment où chacun va donner sa façon de résoudre le problème et la confronter à celles mises en oeuvre par les autres. L'apport des enfants du groupe, les échanges entre pairs, sont des moyens pour saisir la logique des enfants. Dans cet atelier, la présence du maître garantit une évolution positive de l'écriture de chacun vers l'écriture de tous.

Revenons à un exemple concret. Yannick a écrit comme légende de son dessin :

La maîtresse, à qui il a donné sa production, lit l'histoire : OISEAUCHERCHELESOEUFS.

Yannick n'est pas d'accord, il dit qu'il a écrit : L'OISEAU... La maitresse montre le texte de Yannick aux autres enfants du groupe. Ozlem signale immédiatement que tout est attaché. Les deux problèmes posés par le texte de l'enfant sont maintenant clairement posés. Les enfants et la maîtresse vont s'activer pour les résoudre. La maitresse insiste en répétant qu'elle lit OISEAU et pas L'OISEAU et précise qu'il manque quelque chose... 
quelque chose qu'on entend et qui n'est pas écrit. La recherche dans le dictionnaire permet de retrouver rapidement la fiche "UN OISEAU". La lecture de cette fiche répétée par les enfants entraîne l'identification plus précise de UN et de OISEAU et montre que, en effet, Yannick n'a pas écrit L'OISEAU.

Yannick est invité à dire encore une fois ce qu'il a voulu écrire, puis la maîtresse demande l'attention de tous en les priant de dire ce qu'on entend au commencement, et elle répète plusieurs fois L'OISEAU... L'OISEAU... L'OISEAU. Comme elle n'obtient pas de réponse positive, elle donne sa solution en expliquant qu'elle entend la lettre "L" et que c'est pour cela qu'elle l'écrit.

Le deuxième problème est plus rapidement résolu, car la recherche dans le dictionnaire et sur les panneaux de la classe amène les enfants à une reconnaissance de la forme des mots et de leurs limites.

$\mathrm{Au}$ cours du déroulement de ce travail la maitresse a écrit, sur la feuille de Yannick, le texte dans sa forme normale. Elle la montre et la lit aux enfants,

\section{LA FILLE P LES POMMES.}

puis la rend à son auteur.

Le texte d'Ozlem dont nous avons déjà parlé plus haut est plus facile à aborder.

La maîtresse demande à l'enfant de dire son histoire. Celle-ci l'énonce clairement :

\section{"LA FILLE PREND LES POMMES"}

et indique immédiatement qu'elle ne sait pas écrire "PREND". Elle dit aussi qu'elle a mis "P" parce qu'elle l'entend. Le même procédé que celui mis en œuvre pour "L'OISEAU" du texte de Yannick, va permettre de trouver ce que nous entendons après "P". Ici, le "R" est identifié par les enfants. Pourquoi ont-ils trouvé le " $R$ " et pas le "L" alors que la première lettre est souvent plus facile à découvrir? Cette question restera sans réponse. Le reste du mot est donné par la maîtresse.

Nous pourrions multiplier les exemples de ce type qui montrent comment on peut s'appuyer sur la production des enfants et sur leurs erreurs pour les aider à avancer dans la connaissance de l'écrit.

Il est clair qu'il ne s'agit pas de mettre en place un enseignement systématique, et le terme "d'atelier d'accompagnement" est suffisamment explicite pour dire que c'est l'enfant qui est au centre de cette activité. Dans la même journée, la maîtresse risque d'être confrontée à des problèmes très différents en fonction du niveau des enfants.

Par ailleurs, nous voyons très bien que cet atelier est un lieu privilégié de l'évaluation du savoir des enfants, en même temps qu'il est une source abondante d'idées dans la recherche de situations à leur proposer. Même si tous les problèmes posés par les enfants ne peuvent pas être résolus, ils sont au moins identifiés et, de ce fait, ils amènent l'enseignant à mieux cerner sa connaissance des enfants.

48 A la fin de la séquence chaque enfant est en possession de son texte qui se présente maintenant sous une forme normée. 


\section{Atelier autonome}

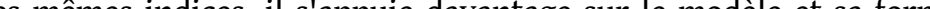
graphique et il repère l'erreur plus rapidement. C'est lui qui montre à Jordan où ce n'est pas pareil.

61 Cette erreur faite par Jordan est assez courante, elle résulte de la contraction de deux mots voisins ayant des formes proches. L'enfant a commencé FOR(ET) puis dans son va-et- 
vient entre le modèle, l'écran et le clavier, il a enchaîné sur (FO)NT. Dans cet exemple la présence du "R" montre qu'il n'a pas sauté le mot "FORET", mais qu'il s'est appuyé sur la partie commune du début des mots (FO) pour se repérer dans le modèle.

Une fois le texte inscrit sur l'écran, il sera imprimé. Ainsi en fin de séquence, l'enfant dispose de sa production dans une forme quasi parfaite.

\section{DISCUSSION}

L'originalité de ce travail tient au fait que l'écriture productive est demandée aux enfants avant qu'ils ne soient en mesure de donner une production normée et que cela se fait dans un contexte de communication. La démarche ainsi appliquée permet à l'enfant, placé en situation d'autonomie, de produire l'écrit à partir de son savoir. Ce savoir prend son origine dans la scolarité antérieure, mais aussi dans ses expériences personnelles, ses observations quotidiennes... C'est une démarche constructiviste, dans laquelle l'enseignant s'adapte à l'enfant et lui propose des situations et des activités qui lui permettent de réfléchir sur le fonctionnement et l'utilisation de la langue écrite. L'enfant crée de l'écrit comme il en reçoit. Il est donc à même d'émettre des hypothèses sur l'origine de cet objet de connaissance et de mieux le comprendre.

64 A la question de savoir si ce travail constructif accompli par l'enfant lui apporte une meilleure connaissance de la langue écrite, nous répondrons affirmativement.

Plusieurs évaluations ponctuelles donnent des résultats tout à fait positifs aux différents niveaux du cycle 2.

En fin de grande section d'école maternelle, une étude menée sur 74 enfants "expérimentaux" et "témoins" répartis dans des classes présentant des caractéristiques socio-culturelles voisines, montre la supériorité des élèves qui ont suivi notre démarche. Cette supériorité est mesurée, à partir d'une épreuve d'écriture inventée, sur 12 critères témoignant du niveau de construction de la langue écrite des enfants. En fin de cours préparatoire et en fin de cours élémentaire les analyses des résultats de plus de 150 enfants évalués à l'aide de diverses épreuves mesurant l'acquisition de la lecture et de l'écriture montrent que l'acquisition de la langue écrite par les élèves ayant suivi la démarche débouche sur un apprentissage différent. Cet apprentissage intègre davantage les éléments servant à la production d'un récit signifiant pour une meilleure communication à autrui (en écriture) ou une meilleure prise de décision (en lecture).

Ces résultats sont partiels, car la démarche est difficile à évaluer globalement, mais ils sont très encourageants. Ils montrent que la prise en compte précoce de l'écriture productive des enfants dans le cadre général d'une conception constructiviste de l'apprentissage et de l'enseignement de la langue écrite est une démarche pouvant s'avérer fructueuse. 
BIBLIOGRAPHIE

\section{Bibliographie}

BESSE, J.M., "De l'écriture productrice à la psychogenèse de la langue écrite", in L'enfant apprenti lecteur, CRESAS, INRP, L'Harmattan, 1993.

FERREIRO, E., GOMEZ PALACIO, M., Lire-écrire à l'école : comment s'y prennent-ils ? CRDP, Lyon, 1988.

FIJALKOW J., "Entrer dans l'écrit : des niveaux successifs." in L'enfant apprenti lecteur, CRESAS, INRP, L'Harmattan, 1993.

FIJALKOW J., FIJALKOW, E., “L'écriture inventée au cycle des apprentissages : étude génétique.” in Les dossiers de l'éducation, $n^{\circ} 18,1991$.

\section{RÉSUMÉS}

Faire écrire des jeunes enfants, c'est leur donner le moyen d'exprimer leurs représentations de la langue écrite dans un contexte de socialisation et de responsabilité. Les ateliers d'écriture, tels que nous les concevons, prennent en compte ces différents aspects pour que l'enfant scripteur construise, dans sa globalité, un langage écrit efficace.

To have the young children write, it's to give them the possibility to express the representation of the writing language inside a context of responsibility and socialisation. The writing laboratory, as we conceive, takes in facts this differents aspects that the script child builds in his globality, an efficace writing language.

\section{INDEX}

Mots-clés : autonomie, constructivisme, écriture, socialisation

Keywords : autonomous, constructiveness, writing

\section{AUTEUR}

JACQUY ALBERT

EURED-CREFI, université de Toulouse - Le Mirail 